\title{
SPIRAL2 at GANIL: \\ A world leading ISOL facility \\ at the dawn of the next decade
}

\author{
S. Gales ${ }^{1}$ \\ ${ }^{1}$ GANIL (DSM-CEA/IN2P3-CNRS), \\ Blvd. H. Becquerel, BP55021, F-14076 Caen cédex5, France
}

November 23, 2006

\begin{abstract}
To pursue the investigation of a new territory of nuclei with extreme N/Z called " terra incognita" several projects, all aiming at the increase by several orders of magnitude of the RIB intensities are now under discussions worldwide. In Europe, two major new projects have been approved recently FAIR@GSI using the so-called "in-fligth" method and SPIRAL2@GANIL,based on the ISOL method. The main goal of SPIRAL2 is clearly to extend our knowledge of the limit of existence and the structure of nuclei deeply in the medium and heavy mass region $(\mathrm{A}=60$ to 140) which is to day an almost unexplored continent. SPIRAL 2 is based on a high power, CW, superconducting driver LINAC, delivering $5 \mathrm{~mA}$ of deuteron beams at $40 \mathrm{MeV}(200 \mathrm{KW})$ directed on a $\mathrm{C}$ converter+ Uranium target and producing therefore more $10^{13}$ fissions $/ \mathrm{s}$. The expected radioactive beams intensities for exotic species in the mass range from $\mathrm{A}=60$ to $\mathrm{A}=140$, of the order of $10^{6}$ to $10^{10}$ pps will surpass by two order of magnitude any existing facilities in the world. These unstable atoms will be available at energies between few $\mathrm{KeV} / \mathrm{n}$ to $15 \mathrm{MeV} / \mathrm{n}$. The same driver will accelerate high intensity $(100 \mu \mathrm{A}$ to $1 \mathrm{~mA})$, heavier ions up to Ar at $14 \mathrm{MeV} / \mathrm{n}$ producing also proton rich exotic nuclei. In applied areas SPIRAL2 is considered as a powerful variable energy neutron source, a must to study the impact of nuclear fission and fusion on materials. The intensities of these unstable species are excellent opportunities for new tracers and diagnostics either for solid state, material or for radiobiological science and medicine. The technical design has reached the point where SPIRAL2 is ready for construction. Project status and foreseen schedule will be presented.. Scientific and technical R\&D programs in collaboration with EU and International partners for the facility as well as for the associated innovative new instruments will be discussed.
\end{abstract}

\section{Introduction}

In the recent years, Rare Isotope Beams (RIB), i.e. beams of synthesized radioactive isotopes, have been recognized by the international scientific community as one of the most promising avenue for the development of fundamental nuclear physics and astrophysics, as well as in applications of nuclear science. The importance of the RIB's has been underlined by NuPECC (Nuclear Physics European Collaboration Committee - an expert committee of the European Science Foundation) which recommend the construction of two new-generation complementary RIB facilities in Europe. One based on the inflight fragmentation (IFF) as proposed for the FAIR facility at GSI (Darmstadt, Germany) and the 
other on isotope-separation on-line (ISOL) method. In ISOL method, a thick target is bombarded with a primary light or heavy-ion beam or with a secondary neutron beam in case of fission targets. The produced radioactive nuclei are post-accelerated to energies from keV to tens of MeV. The ISOL method provides high-intensity beams of high optical quality comparable to stable beams. The ultimategeneration RIB ISOL facility will be realised when the EURISOL facility will be built. However, a full engineering design study and necessary R\&D programme lead to the expected beginning of operation of the EURISOL facility around 2020. Because of the time-line for EURISOL, NuPECC recommends the construction of intermediate-generation facilities that will benefit the EURISOL project in terms of R\&D and that will give the community opportunities to perform research and applications with RIB. Among the proposed intermediate facilities SPIRAL2 at GANIL (Caen, France), meets in terms of physics potential, site and size of the investments, the criteria of European dimension.

In March 2005, the ESFRI (European Strategy Forum on Research Infrastructures) published the "List of Opportunities", which includes 23 Research Infrastructure projects corresponding to major needs of the European scientific community in the coming years. The FAIR and SPIRAL 2 are among the selected projects and were recommended as Research Infrastructures necessary to maintain Europe's position at the cutting-edge of world research.

The French Ministry of Research took a decision on the construction of SPIRAL 2 in the end of May 2005. Its construction cost, estimated to 130 Meuros (including personnel and contingency), will be shared by French founding agencies CNRS/IN2P3 and CEA/DSM, the Region of Basse Normandie and the European partners. The construction will last about six years and operation of the facility will cost 8.5 Meuros per year.

The investments costs amount to 95 Meuros and personnel costs to 35 Meuros, for the construction period 2006-2012. Funding from EU 7th framework and from others partnership countries are expected to contribute for about $20 \%$ to this budget.

The first beams are expected by the end of 2011. As an added bonus, the facility will also produce over $10^{15}$ neutrons per second, making it the world's most powerful source of fast neutrons for several years.

The facility is an extension to the SPIRAL 1 device, which opened five years ago at GANIL. SPIRAL 2 will reinforce European leadership in the field of exotic nuclei and will serve a community of about 600 scientists.

\section{Description of the project}

The quest for Rare Isotope Beams (RIB), which are orders of magnitude more intense than those currently available, is the main motivation behind the SPIRAL 2 project.

The new SPIRAL2 facility is a major improvement of the SPIRAL1 facility. More than 20 laboratories from 10 countries from 2001 to 2005 have developed the conceptual and the technical design of SPIRAL2. More than 200 scientists and engineers have contributed to the scientific case and the design of the facility1).

The SPIRAL 2 facility (figure 1) is based on a high power, superconducting driver LINAC, which will deliver a high intensity, $40 \mathrm{MeV}$ deuteron beam as well as a variety of heavy-ion beams with mass over charge ratio equals to 3 and energy up to $14.5 \mathrm{MeV} /$ nucl.. Using a carbon converter, the $5 \mathrm{~mA}$ deuteron beam and a uranium carbide target, a fast-neutron induced fission is expected to reach a rate of up to $10^{14}$ fissions/s. The RIB intensities in the mass range from $A=60$ to $A=140$ will surpass by one or two order of magnitude any existing facilities in the world. A direct irradiation of the UCx target with beams of deuterons, ${ }^{3,4} \mathrm{He},{ }^{6,7} \mathrm{Li}$, or ${ }^{12} \mathrm{C}$ can be used if higher excitation energy leads to higher production rate for a nucleus of interest.

The neutron-rich fission RIB could be complemented by beams of nuclei near the proton drip line 


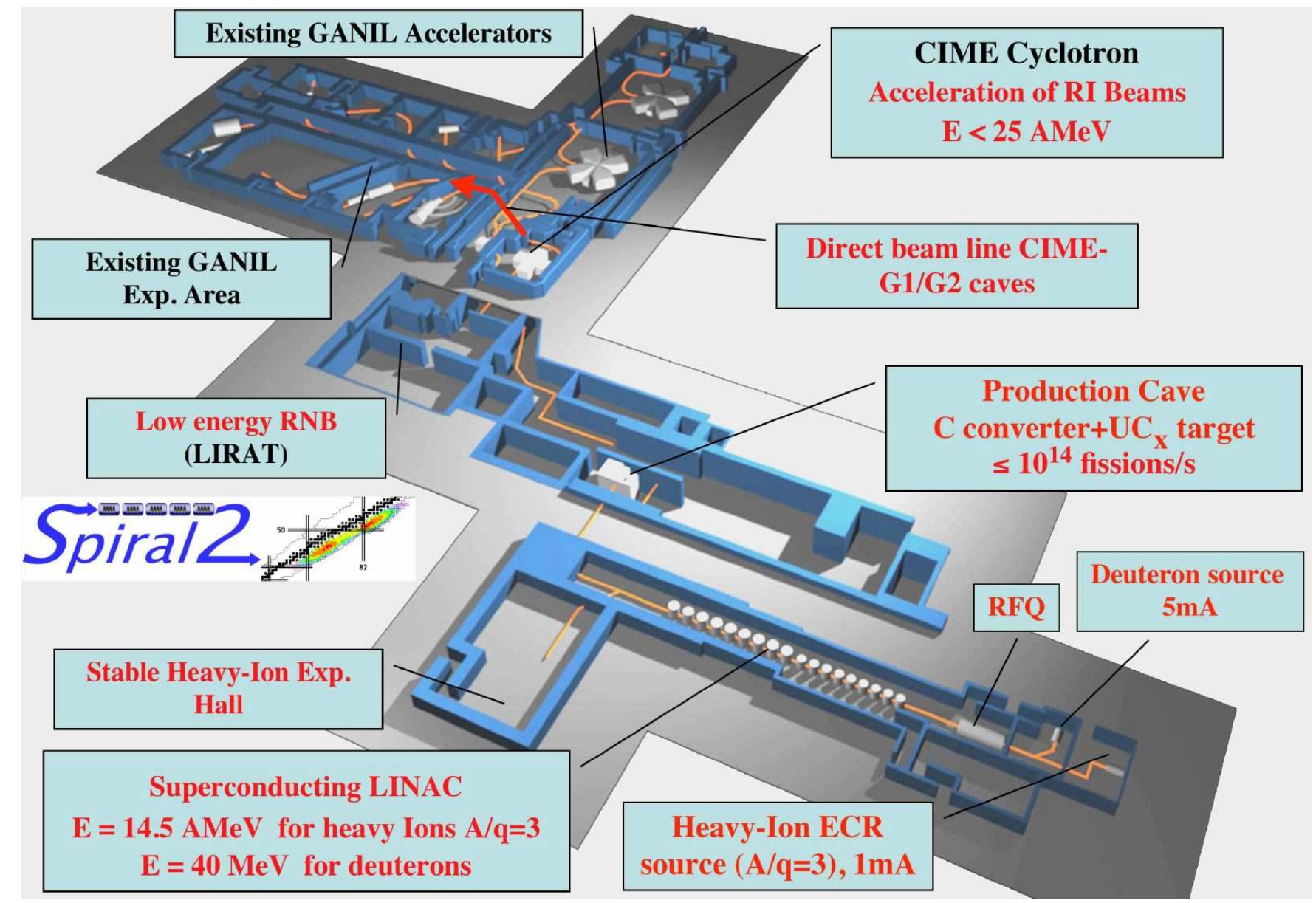

Figure 1: Schematic layout of the SPIRAL 2 facility at GANIL

provided by fusion-evaporation or transfer reactions. For example, a production of up to $8 \times 10^{4}$ atoms of ${ }^{80} \mathrm{Zr}$ per second using a $200 \mu \mathrm{A}{ }^{24} M g^{8+}$ beam on a ${ }^{58} N i$ target should be possible. Similarly, the heavyand light-ion beams from LINAG on different production targets can be used to produce high-intensity light RIB with ISOL technique.

The extracted RIB will be subsequently accelerated to energies of up to $20 \mathrm{MeV} /$ nucl. (typically 6-7 MeV/nucl. for fission fragments) by the existing CIME cyclotron. Thus using different production mechanisms and techniques SPIRAL 2 would allow performing experiments in a wide range of neutronand proton-rich nuclei far from the line of stability (figure 2).

One of the important features of the future GANIL/SPIRAL/SPIRAL 2 facility will be a possibility to deliver up to three stable or radioactive beams simultaneously in the energy range from keV to several tens of $\mathrm{MeV} /$ nucl..

SPIRAL2 has also a remarkable potential for neutron-based research both for fundamental physics and various applications. In particular, in the neutron energy range from a few MeV to about 35 $\mathrm{MeV}$ this research would have a leading position for the next 10-15 years if compared to other neutron facilities in operation or under construction worldwide. 


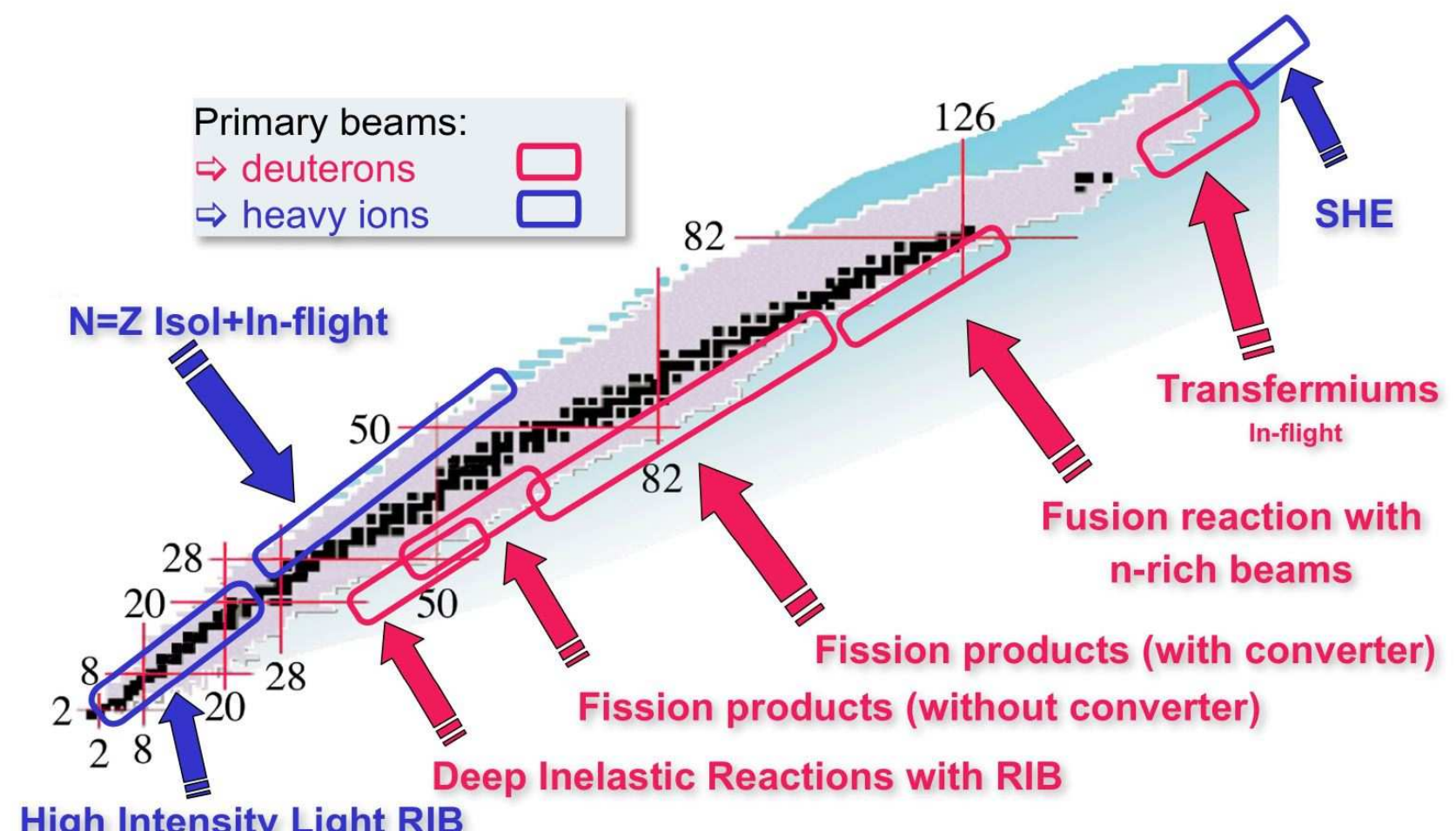

High Intensity Light RIB

Figure 2: Regions of the chart of nuclei accessible for research of nuclei far from stability at SPIRAL2.

\section{Scientific opportunities and performances}

Very intense stable heavy ions beams will be available at the exit of the LINAG accelerator. These heavy ions (from He to $\mathrm{Ca}$ in a first step, then up to $\mathrm{U}$ ), will reach intensities from $1 \mathrm{p} \mu \mathrm{A}$ up to $1 \mathrm{pmA}$ using new PHOENIX ion sources in combination with $\mathrm{A} / \mathrm{Q}=1 / 3 \mathrm{RFQ}$ injector and later $\mathrm{A} / \mathrm{Q}=1 / 6$ for heavier ions (Kr to U) as illustrated in Fig.3.

Reaching such beams intensities will open a wide range of physics experiments not accessible to day. Super Heavy Elements synthesis and spectroscopy, spectroscopy at or beyond the proton drip line, multinucleon transfer and deep inelastic scattering, study of mechanisms and reaction product distributions, production and study of isomers ,Ground state properties of rare nuclei.

A new device, S3 (for Super Separator Spectrometer) has been proposed for experiments with the very high intensity stable beams of SPIRAL2 coming from the LINAG accelerator1).

Most of the physics cases listed above can be seen as either the study of rare processes or as the study of a secondary reaction with exotic ions produced in a first step reaction.

S3 shall be designed to answer most experimental needs of these physics cases. The crucial point of the device is to cope with the very high intensity beams provided by the LINAG accelerator. That means:

-the targets should be able to cope with very high power dissipation,

-the reaction products should be separated from the beam ions before they can be detected,

-the full optics of the spectrometer should reduce the background coming from scattered ions and activation products, 


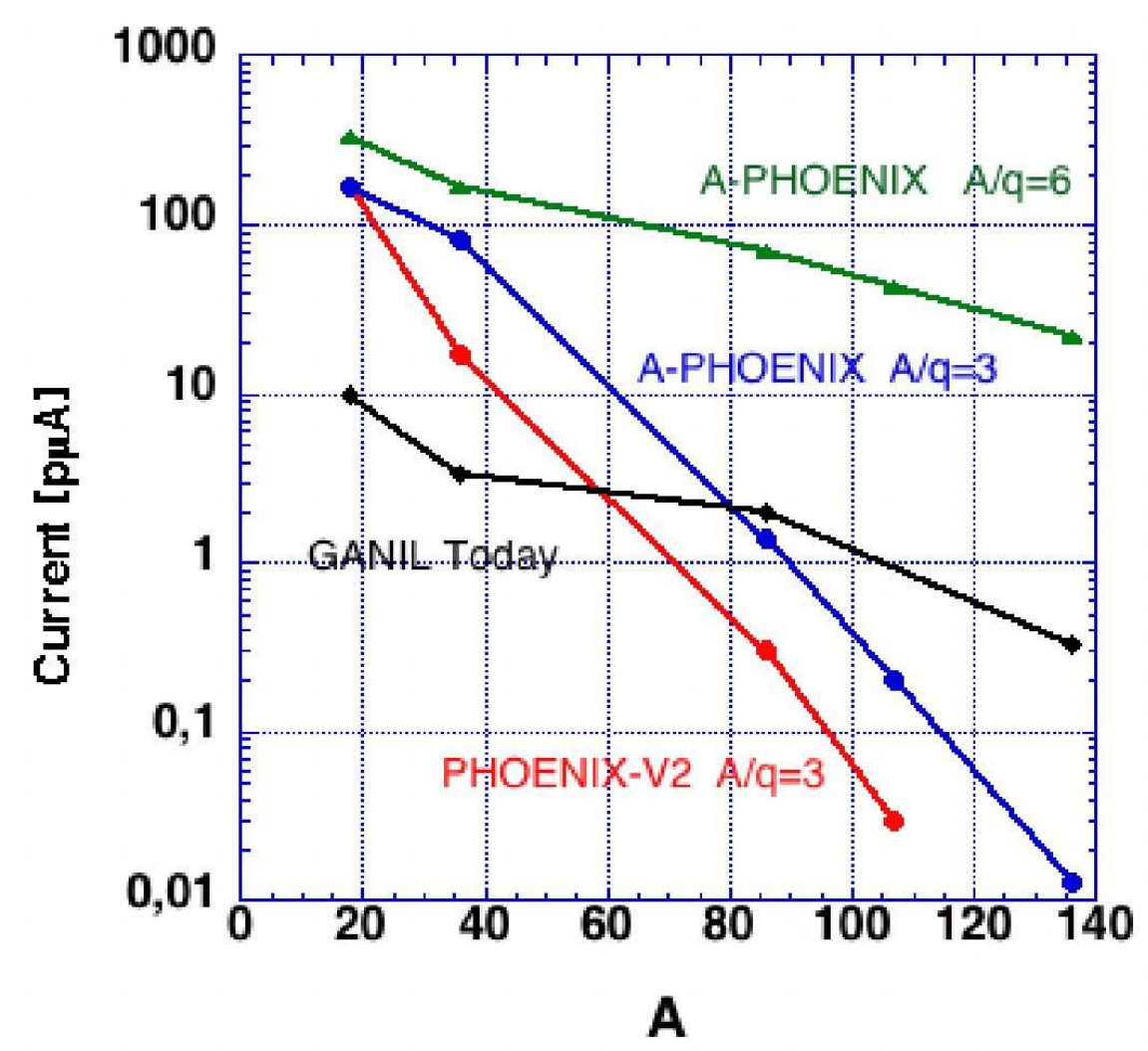

Figure 3: Expected intensities of stable heavy ion beams at the exit of the LINAG accelerator for various versions of PHOENIX ion sources.

Meanwhile, the vast array of different physics that could be possible with S3 implies that it should be compatible with various detection settings. The basic design considered could be the following (see figure 3):

The primary beam will impinges on a primary target which should be made resistant to the high fluxes (rotating cooled targets, gas or liquid targets...)

The separation of the beam can proceed in two steps, the first being a high rejection device and an appropriate beam stopper.

After this first step, ions of interest could, if necessary, react on a secondary target. As they deal with moderate fluxes, this secondary target point could have gamma or particle prompt detection.

Next to this point, a secondary spectrometer helps with separation if very high purity is needed. It has some mass/momentum resolution and could rotate for reactions products at large angles.

The final focal plane can be equipped with the relevant detection system.

The SPIRAL2 facility at GANIL will produce radioactive isotopes ranging from the lightest to very heavy elements beyond uranium. Different production mechanisms will be utilised: fission of ${ }^{238} U$ to produce medium-mass, neutron-rich isotopes, fusion evaporation for medium-mass, neutron-deficient nuclei, and transfer and deep-inelastic reactions for light to heavy nuclei closer to the line of stability. Examples of reactions leading to the production of light neutron rich and $\mathrm{N}=\mathrm{Z}$ exotic nuclei are listed in Table. 1 below.

SPIRAL2 beam intensities for fission fragments after acceleration are shown in fig.5. For SPIRAL2 the RIB intensities in the mass range from $A=60$ to $A=140$ will be of the order of $10^{6}$ to $10^{11}$ part./s surpassing by one or two order of magnitude any existing facilities in the world. For example, the intensities should reach, $10^{9}$ part./s for ${ }^{132} \mathrm{Sn}$ and $10^{10} \mathrm{pps}$ for ${ }^{92} \mathrm{Kr}$

The DESIR collaboration, formed after the SPIRAL2 workshop on low-energy physics at GANIL 


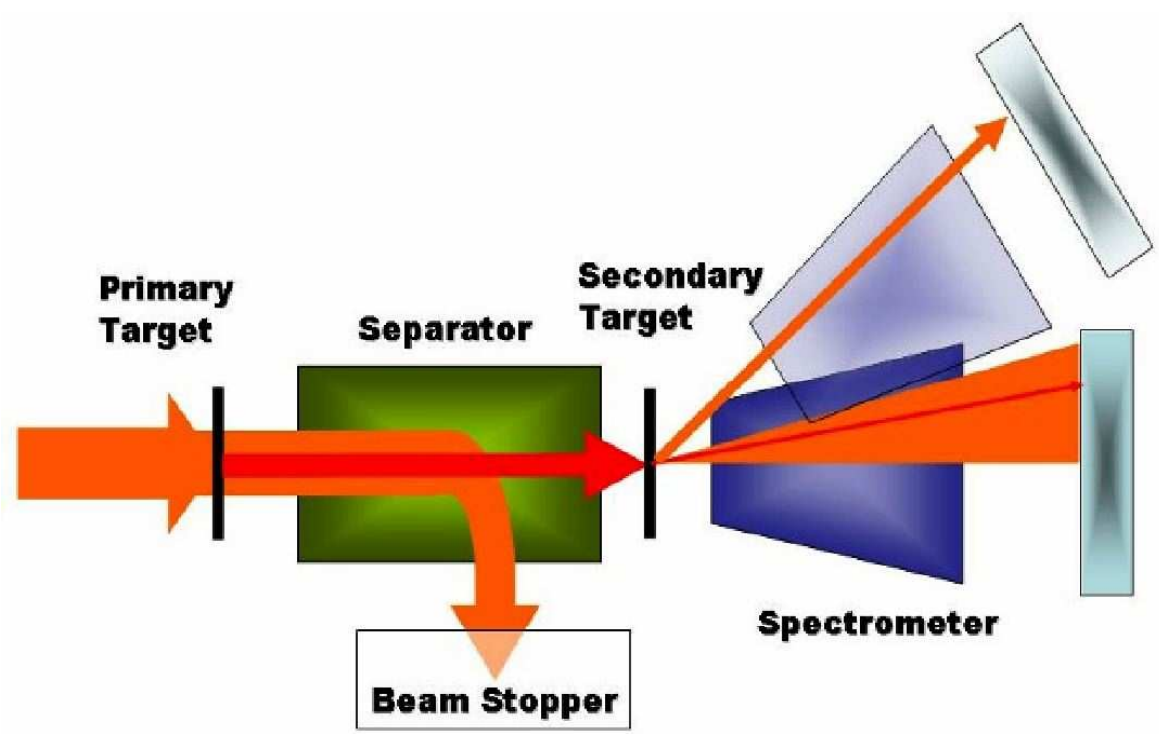

Figure 4: Basic scheme for S3 design

Table 1: default

\begin{tabular}{|c|c|c|c|}
\hline RI Beam & Reaction & Production method & Yield (min. - max.) pps \\
\hline$\overline{{ }^{6} \mathrm{He}}$ & $\overline{{ }^{9} \mathrm{Be}(n, \alpha){ }^{6} \mathrm{He}}$ & $\overline{I S O L}$ & $\overline{5 x 10^{7}-10^{12}}$ \\
\hline${ }^{1} 1 C$ & ${ }^{14 N}(p, \alpha)^{11} C$ & $I S O L$ & $10^{7}-3 x 10^{11}$ \\
\hline${ }^{1} 5 O$ & ${ }^{15} \mathrm{~N}(d, 2 n){ }^{15} \mathrm{O}$ & $I S O L$ & $3 x 10^{7}-10^{10}$ \\
\hline${ }^{18} \mathrm{Ne}$ & ${ }^{19} \mathrm{~F}(p, 2 n)^{18} \mathrm{Ne}$ & $I S O L$ & $6 x 10^{6}-7 \times 10^{9}$ \\
\hline${ }^{34} \mathrm{Ar}$ & $\left.{ }^{35} C l(p, 2 n)\right)^{34} A r$ & $I S O L$ & $2 x 10^{6}-2 x 10^{8}$ \\
\hline${ }^{56} \mathrm{Ni}$ & ${ }^{58} N i(p, p 2 n)^{56} N i$ & Batchmode & $2 x 10^{4}-10^{8}$ \\
\hline${ }^{58} \mathrm{Cu}$ & ${ }^{58} \mathrm{Ni}(p, n){ }^{58} \mathrm{Cu}$ & Batchmode & $10^{4}-10^{8}$ \\
\hline${ }^{80} \mathrm{Zn}$ & ${ }^{24} \mathrm{Mg}+{ }^{58} \mathrm{Ni}$ & In - flight & $<3 x 10^{4}$ \\
\hline
\end{tabular}

Table 2: Calculated production yields for light and $\mathrm{N}=\mathrm{Z}$ exotic nuclei using transfer or fusion evaporation reactions

in July 2005, proposes the construction of an experimental facility to exploit the low-energy "exotic" beams from SPIRAL2. Experiments performed at other ISOL facilities (e.g., ISOLDE or ISAC) show that a high degree of purity is necessary for the ions beams. The most efficient and universal way of achieving isotopically pure beams is a high-resolution mass separator.

At SPIRAL2 and its low-energy beam facility DESIR, there is an opportunity of building three kinds of setup to try to cover the whole nuclear chart. A $\beta$-NMR setup will be suitable to study precisely the moments of key cases and, in combination with collinear hyperfine spectroscopy, get unambiguous spin assignments. With a collinear spectroscopy setup, we will have the possibility to study series of isotopes in the intermediate and heavy mass regions. For the heavy elements, a double laser $+\mathrm{RF}$ spectroscopy in a Paul trap will be used to study the hyperfine anomaly and higher-order moments up to very high precision.

Two main regions of the mass chart in the vicinity of the $\mathrm{N}=50$, and 82 shell closures are of special interest

1) The $\mathrm{N}=50$ region: 


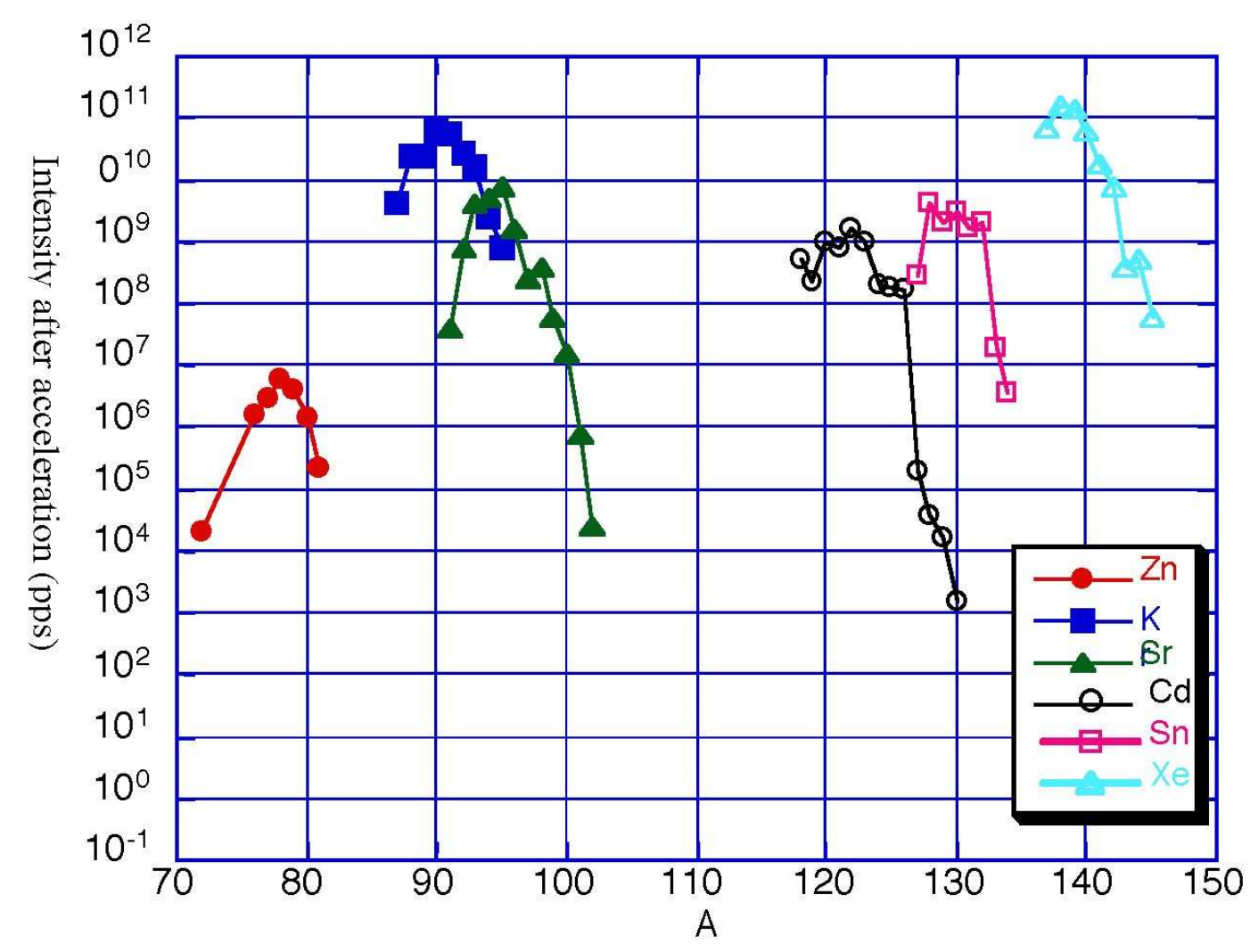

Figure 5: Fission fragments beam intensities from SPIRAL2 after acceleration

One important issue is to determine how thick can be the neutron skin for nuclei far from stability? From the measurement of the charge radius, one has access to the neutron radius. A key element would be germanium which is produced in large quantities in neutron stars. So the measurements of the charge radius change of this element and its neighbours (Zn, As, Ga, Se) over the magic shell N=50 using collinear laser spectroscopy will answer this question.

Another typical example of an element that has to be studied is of course nickel as close as possible to ${ }^{78} \mathrm{Ni}$. How can the charge radius evolve so far from stability? Is this doubly-magic nucleus still spherical?

2) The $\mathrm{N}=82$ region:

For all the studied elements between tellurium $(Z=52)$ and samarium $(Z=62)$, the charge radius change exhibit a kink at $N=82$ showing an increase in deformation beyond this magic number. The interesting feature is that the more one approaches tin $(\mathrm{Z}=50)$ the less pronounced is the kink. A lot of nuclear models predict that no kink is present at $\mathrm{N}=82$ for tin, a fact which should be a signature of a neutron skin for the very neutron-rich isotopes. Studying tin very far from stability will be a very stringent test for these nuclear models. In contrast, indium $(Z=49)$, iodine $(Z=53)$ and cadmium $(Z=48)$ should present interesting kinks at $N=82$. Silver isotopes $(Z=48)$ have been studied until $A=110$. This element is the heaviest in this region to show a sizeable shape change at $N=60$. The heaviest one could have interesting shapes.

Nuclei were created during major events that made the evolution of the universe and are the witness of the origin of matter: while the lightest elements were created in the first minutes following the big bang, heavier ones are synthesized in stars. Understanding our world, its origin and its evolution is one goal of nuclear physics. However, these various nucleosynthesis processes involve radioactive nuclei. Due to their short life-time most of them have not survived long enough to be present on earth. To be 
studied those "exotic" nuclei must be synthesized in laboratories. SPIRAL 2 will abundantly produce some of them opening new investigation field.

The future SPIRAL2 facility will contribute prominently to several areas of active research in nuclear astrophysics, such as explosive hydrogen burning, s-process and r-process nucleosynthesis, which are linked to astrophysical observations (novae, X-ray bursts, type 2 supernovae,etc...).

For example, radiative capture cross-sections can be determined from spectroscopic factors (or Asymptotic Normalization Coefficients) obtained in transfer reaction or elastic resonant scattering. From a general point of view, detailed spectroscopy of the nuclei of interest is crucial to insure efficient application of indirect methods, which can be obtained by using these two reactions.

Neutron capture cross-sections are often calculated in the framework of the statistical HauserFeschbach model, which assumes the presence of a high density of states above the neutron separation energy with various spin and parity values. The use of this statistical approach is not appropriate to calculate neutron capture cross sections for neutron-rich at closed shell, since the neutron-separation energy is small and the level density low. In such cases, the main contribution is obtained from direct captures on few bound states, mainly of $\mathrm{s}$ or $\mathrm{p}$ waves, or/and by resonance capture slightly above the neutron-energy threshold.

The $(\mathrm{d}, \mathrm{p})$ reaction can be used to simulate the $(\mathrm{n}, \alpha)$ capture on medium-mass nuclei at SPIRAL2 near the r-process possible paths .

The way the energy of theses s or p states evolve from $Z=50$ down to $Z=40$ determines how neutron capture cross sections will evolve along the $\mathrm{N}=82$ shell closure and how the $\mathrm{r}$ process synthesis of nuclei will be blocked at this shell closure. So again, the evolution of these p states and the underlying physics which makes them move with respect to other orbits is an essential ingredient to model the $\mathrm{r}$ process. The neutron rich isotopes of Sn and Cd nuclei are excellent test cases.

The neutron-capture through the soft Pigmy resonance could also play an important role if the energy of the soft pigmy vibration mode is peaked around the Sn value. So far some models predict this resonance to lie around $4 \mathrm{MeV}$, whereas recent experiment in the $\mathrm{Sn}$ isotopic chain show that this resonance is peaked at higher energy. The way this energy is shifted when moving from Sn to Cd isotopic is certainly an important issue to consider. Since the direct measurements of neutron-capture cross sections on very unstable nuclei is technically not feasible their determination should be provided by models, constrained by spectroscopic information (energy, spin, spectroscopic factors of the bound and unbound levels in the $\mathrm{A}+1$ nucleus) obtained by $(\mathrm{d}, \mathrm{p})$ transfer reactions.

The scientific opportunities discussed above are just a few examples among many exciting avenues which can be investigated with SPIRAL2.

New exotic shapes and excitation modes, halo-like and molecular structures, single particle properties and new magic numbers, new modes of nuclear decay have been recently observed, while studies of fundamental symmetries, testing and refinement of the Standard Model and of fundamental interactions in very exotic nuclei have all a huge potential of discovery.

In addition, let us stress once more time that, thanks to the high energy and high intensity neutron flux available at SPIRAL2, the facility will offer a unique opportunity for material irradiations and cross-section measurements both for fission (notably accelerator driven systems (ADS) and Gen-IV fast reactors) and fusion related research, tests of various detection systems and of resistance of electronics components to irradiations, etc.

\section{Construction of the facility and International Collabora- tions}

Since September 2005, a project construction group has been formed and the whole project has been organized around three main sections - The driver accelerator - The secondary radioactive beams (pro- 


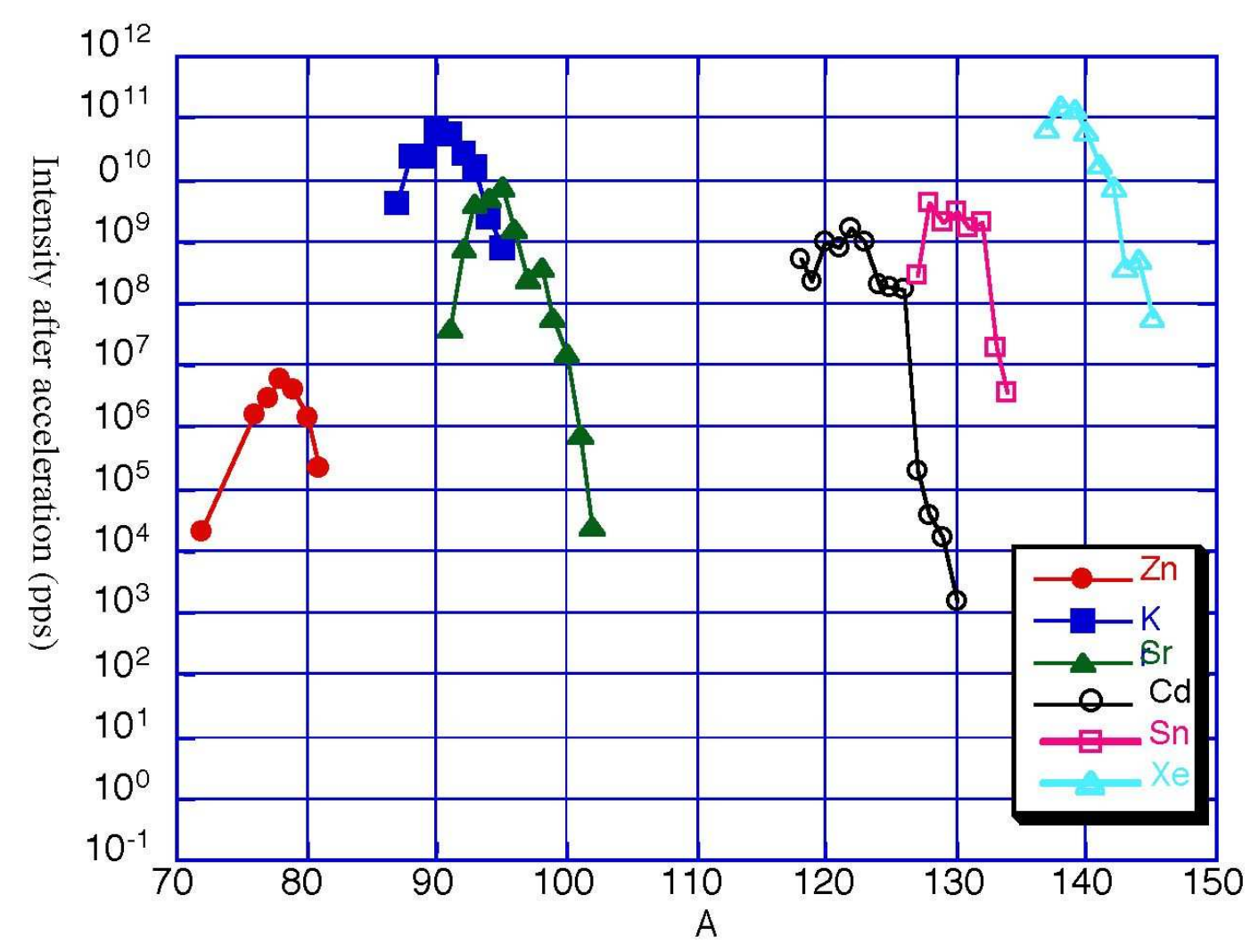

Figure 6: SPIRAL2 driver accelerator divided into main components

duction and transport) - The infrastructure (buildings, vacuum and cryogenics, power supplies) The project has been divided in each section into work packages. Examples of work packages related to the driver accelerator are shown in Figure 6.

For the driver accelerator, most of the work packages are under the responsibility of IPN Orsay and DAPNIA/DSM/Saclay laboratories. The R\&D on prototypes' for the RFQ, SC cavities and RF couplers are completed and order of driver components will be completed at the end of this year

A SPIRAL2-France consortium had been set to coordinate the efforts of 9 French nuclear physics laboratories from CNRS and CEA.

Moreover SPIRAL2 facility will be built by a large European and International collaboration.

Several MoU have been already signed or are in final negotiation stages with JINR Dubna, Israel Roumania, Isolde (CERN), Italy, UK ,Germany,Poland,Canada,Japan and US ,both for accelerator components (control system, beam loss monitors, beam lines) and/or construction of components of the RIB production units (neutron converter , beam lines, target-ions sources ,laser ion sources, highresolution magnetic separator ,....).

At the EU research organisation level, SPIRAL2 as well as FAIR have been selected by the ESFRI

Committee and a proposal for the construction phase in the framework of the 7th FPRD is under preparation.

The process of formation of international collaborations around the SPIRAL 2 research programme consists of:

- 5 specialised workshops held in 2004-2006 under umbrella of the Scientific Advisory Committee of SPIRAL 2:

o Neutrons for science at SPIRAL 2, 13-14/12/2004; 
o Physics with separated low energy beams at SPIRAL2, 4-5/07/2005;

o Future prospects for high resolution gamma spectroscopy at GANIL, 4-6/10/2005;

o Nuclear Astrophysics with SPIRAL2, 17-18/10/2005;

o SPIRAL2 Reactions, 19-21/10/2005;

Two other workshops on interdisciplinary research and theoretical aspects will be held in the end of 2006/beginning of 2007;

- Call for letters of intent in March 2006 and their evaluation by October 2006;

- Call for proposals in Spring-Summer 2007.

In terms of schedule for the new facility, the ambition is to start operation of the driver accelerator with intense stable beams before the end of 2011 and to complete the whole project including RIB production and post acceleration of fission fragments before the beginning of 2013.

\section{Conclusion}

Nuclear physics has been revolutionized by the recent development of the ability to produce accelerated beams of radioactive nuclei. For the first time it will be possible to study reactions to produce the 6000 to 7000 nuclei we believe exist rather than the 300 stable ones that nature provides. At the larger scale one wants to understand the limits of nuclear stability by producing exotic nuclei with vastly different numbers of neutrons and protons.

SPIRAL2 is major expansion of the SPIRAL facility at GANIL, which will help maintain European leadership in ISOL (Isotope separation on line) development, aiming at two orders of magnitude increase of the secondary beams available for nuclear physics studies.

The technical challenges of the acceleration, targetry and experimental equipment will provide essential knowledge on the road toward EURISOL; the most advanced nuclear physics research facility presently imaginable and based on the ISOL principle. It is expected, that the realisation of SPIRAL2 will substantially increase the know-how of technical solutions to be applied not only for EURISOL but also in a number of other European/ world projects. The scientific programme, proposes the investigation of the most challenging nuclear and astrophysics questions aiming at the deeper understanding of the nature of matter.

SPIRAL2 will contribute to the physics of nuclear fission and fusion based on the collection of unprecedented detailed basic nuclear data, to the production of rare radioisotopes for medicine, to radiobiology and to material science.

\section{References}

[1] www.ganil.fr/research/developments/spiral2/ 